WU-B 00-09

May, 302000

hep-ph/0005318

\title{
Large momentum transfer electroproduction of mesons
}

\author{
Han Wen Huang and Peter Kroll \\ Fachbereich Physik, Universität Wuppertal, 42097 Wuppertal, Germany
}

\begin{abstract}
Assuming the proton's light-cone wave function to be dominated by small parton virtualities and small intrinsic transverse momenta, we show that the electroproduction amplitudes at large momentum transfer factorize into parton-level subprocess amplitudes and form factors representing $1 / x$ moments of skewed parton distributions. On the basis of a wave function overlap model for the form factors we present detailed predictions for the electroproduction cross sections. We also comment on large momentum transfer photoproduction.
\end{abstract}




\section{Introduction}

The interest in hard exclusive reactions has recently been renewed in the context of skewed parton distributions (SPDs) [1, 2, 3]. The SPDs, defined as hadronic matrix elements of bilocal products of quark or gluon field operators, are hybrid objects which combine properties of form factors and ordinary parton distributions. In fact reduction formulas reveal the close connection of these quantities. It has been shown that, at large photon virtuality, $Q^{2}$, and small momentum transfer, deeply virtual Compton scattering (DVCS) [3, 4] and deeply virtual electroproduction of mesons (DVEM) [5, 6] factorize into hard photonparton scattering and SPDs describing the soft coupling between partons and hadrons. DVEM is dominated by longitudinally polarized photons for $Q^{2} \rightarrow \infty$; the cross section for transversally polarized photons is suppressed by $1 / Q^{2}$. Complementary to the large $Q^{2}$ region is the large momentum transfer region (small $Q^{2}$ ). In this kinematical region Compton scattering off protons factorizes into a hard parton-level subprocess and a soft proton matrix element that is described by new form factors [7]. These form factors represent $1 / x$-moments of SPDs at large momentum transfer. Based on light-cone wave function overlaps as a model for the SPDs, detailed predictions for cross sections and polarization observables for real and virtual Compton scattering have been achieved in Refs. [7, 8, 9].

Here, in this work, we are going to apply the soft mechanism proposed in Refs. [7, 8] to electroproduction of flavor neutral pseudoscalar $\left(P=\pi^{0}, \eta, \eta^{\prime}\right)$ and longitudinally polarized vector $\left(V=\rho^{0}, \omega, \phi\right)$ mesons. We will show that all arguments given in Ref. [7] in order to establish factorization of Compton scattering, apply here too. I.e. provided the virtualities of the partons and their intrinsic transverse momenta, defined with respect to their parent proton's momentum, are restricted by the proton's wave function, the dominant contribution to electroproduction is generated from the handbag-type diagram shown in Fig. 1. It factorizes into meson electroproduction off partons and soft proton matrix elements described by the same type of form factors as appear in Compton scattering. It is shown in Ref. [7] that, at large momentum transfer, there is one parton with a large virtuality that couples to the meson and forces the exchange of at least one hard gluon. We, therefore, follow the concept used in the calculation of DVEM [5, 6] and treat meson electroproduction off partons to leading-twist, lowest order perturbative QCD. A purely soft mechanism for large momentum transfer electroproduction of mesons, i.e. a soft overlap of the three light-cone wave functions for the hadrons involved is not possible [7]. It is to be stressed however that the soft mechanism is not dominant for asymptotically large momentum transfer. In this limit the hard perturbative mechanism, for which all partons participate in the hard process, provides the leading contribution [10] and the soft one merely represents a power correction. In this respect factorization of the soft mechanism is not on the same footing as the one, say, for DVEM, where the factorising diagrams are dominant for asymptotically large photon virtuality, and where factorization can be proven to hold in all orders of perturbation theory. The soft mechanism applies to photoproduction of mesons as well. However, the contributions from the hadronic component of the photon seem to dominate these processes for values of energy and momentum transfer accessible in current experiments. 


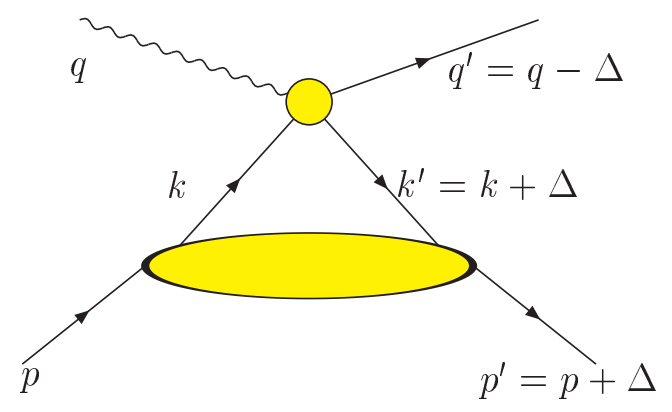

Figure 1: The handbag-type diagram for electroproduction of mesons. The large blob represents a sum over all spectator configuration. $k$ and $k^{\prime}$ denote the momenta of the active partons. The small blob stands for meson electroproduction off partons.

It is also important to realize that the soft mechanism is complementary to the perturbative one, and both the contributions have to be taken into account in principle. However, recent developments, initiated by the CLEO measurements [11] of the $\pi \gamma$ transition form factor and its theoretical analysis, e.g. [12, 13, 14], revealed that soft contributions play an important role in hard exclusive reactions at experimentally accessible momentum transfer which is of the order of a few $\mathrm{GeV}$. Indeed, in the case of the electromagnetic form factor of the proton, the perturbative contribution has been shown to be small as compared to experiment [15, 16] provided the end-point regions, where one of the parton momentum fractions tends to zero, and where perturbative QCD is not applicable [17], are sufficiently suppressed. This can be achieved by employing the modified perturbative approach 18 in which the transverse degrees of freedom and Sudakov suppressions are taken into account.

The soft contribution to large momentum transfer Compton scattering evaluated along the same lines as for the electromagnetic form factors, is in reasonable agreement with experiment [7, 8]. The perturbative contribution, on the other hand, has only been calculated to leading-twist accuracy [19] and is way below the Compton data [20] unless strongly asymmetric, i.e. end-point concentrated distribution amplitudes are used. These give, however, results for which the bulk of the contribution is accumulated in the soft end-point regions where the assumptions of leading-twist perturbative calculations break down. Even if asymmetric distribution amplitudes are utilized one obtains a perturbative contribution to Compton scattering that likely amounts to less than $10 \%$ of the data for momentum transfer in the region of a few $\mathrm{GeV}$ [19]; the onset of the perturbative regime is expected to be above $10 \mathrm{GeV}$. The calculation of the leading-twist perturbative contribution to photoproduction of mesons has been attempted by Farrar et al. [21]. The results are at drastic variance with experiment [20, 22] and need verification since the method for the numerical integrations used by Farrar et al. is questionable and known to fail in Compton scattering. On account of experience with electromagnetic form factors and Compton scattering, we will assume that the soft contribution to electroproduction of mesons are much larger than the perturbative ones for momentum transfers of the order of a few $\mathrm{GeV}$ and that the onset of the perturbative regime is beyond $10 \mathrm{GeV}$. There is still another contribution to 
electroproduction: it has two active partons, the photon couples to one of them while, by insertion of a hard gluon, the other one generates the vector meson. This contribution has the topology of the so-called cat's ears diagrams. It has been discussed in Ref. [7] that, in the large momentum transfer region, large virtualities or intrinsic transverse momenta occur in these diagrams forcing the exchange of additional hard gluons. It is reasonable to assume that the magnitude of the cat's ears contribution is between the soft and the perturbative ones.

The paper is organized as follows: In Sect. 2 we will present the derivation of the soft mechanism. Next we will discuss the necessary phenomenological input that parameterizes the soft hadronic matrix elements (Sect. 3). In Sect. 4 we will comment on the case of photoproduction and then present our results for electroproduction of mesons (Sect. 5). In Sect. 6 we present our summary.

\section{The soft mechanism}

We are interested in electroproduction of mesons in the kinematical region where the Mandelstam variables $s=(p+q)^{2},-t=-\Delta^{2}$ and $-u=-\left(p-q^{\prime}\right)^{2}$ are large on a hadronic scale, $\Lambda$, of order $1 \mathrm{GeV} . Q^{2}$ is not considered as a large scale. Therefore the limit $Q^{2} \rightarrow 0$, the case of photoproduction, is included in the following. The calculation of soft contributions to the process of interest can be performed in full analogy to the case of Compton scattering; all its steps can be adopted straightforwardly. We can, therefore, restrict ourselves to an outline of the calculation; for details we refer to [0]. The process amplitude is evaluated from the handbag-type diagram shown in Fig. 1 where also the four-momenta are defined (as usual $Q^{2}=-q^{2}$ ). We work in a symmetric frame where the transverse momenta of the incoming and outgoing protons are treated in a symmetric way (see Fig. 2)

$$
p=\left[p^{+}, \frac{m^{2}-t / 4}{2 p^{+}},-\frac{1}{2} \boldsymbol{\Delta}_{\perp}\right], \quad p^{\prime}=\left[p^{+}, \frac{m^{2}-t / 4}{2 p^{+}}, \frac{1}{2} \boldsymbol{\Delta}_{\perp}\right]
$$

where $m$ is the mass of the proton. The plus and minus light-cone components of the momentum transfer are zero in this frame $\left(\Delta^{+}=\Delta^{-}=0\right)$ and therefore $t=-\Delta_{\perp}^{2}$. The chief advantage of the symmetric frame is that the skewedness parameter, defined by

$$
\zeta=-\frac{\Delta^{+}}{p^{+}}=1-\frac{p^{\prime}}{p^{+}}
$$

is zero. In order to specify the frame fully we further impose $p_{3}+q_{3}=0$. It coincides with c.m. frame for photoproduction with the 3 -axis along $\mathbf{p}+\mathbf{p}^{\prime}$.

The parton momenta are denoted by $k_{i}$ and $k_{i}^{\prime}$. They are characterized by the usual momentum fractions

$$
x_{i}=k_{i}^{+} / p^{+}, \quad x_{i}^{\prime}=k_{i}^{\prime+} / p^{++},
$$

and the transverse components $\mathbf{k}_{\perp i}$ and $\mathbf{k}_{\perp i}^{\prime}$. Because of $\zeta=0$ in the frame we are working, $x_{i}=x_{i}^{\prime}$. The arguments of the light-cone wave functions are given by the momentum 


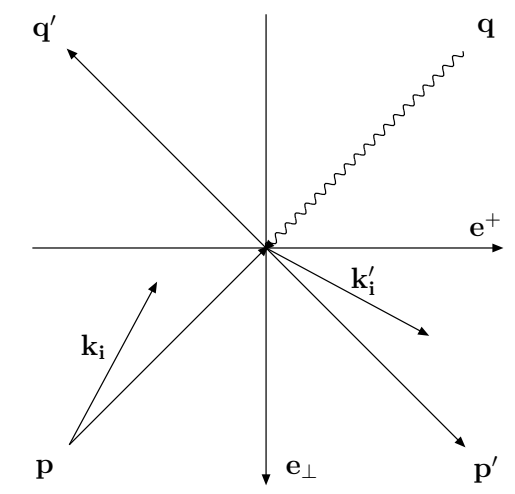

Figure 2: Light-cone plus and transverse components of hadron, photon and parton momenta in the symmetric frame.

fractions and the intrinsic transverse parton momenta, i.e. the transverse components of the parton momenta in a frame where the transverse momentum of the parent proton is zero. By performing appropriate (transverse) boosts one finds for the light-cone wave function arguments of the incoming hadron

$$
\tilde{x}_{i}=x_{i}, \quad \tilde{\mathbf{k}}_{\perp i}=\mathbf{k}_{\perp i}+x_{i} \Delta_{\perp} / 2 .
$$

The arguments of the light-cone wave function of the scattered proton are $\hat{x}^{\prime}=x_{i}^{\prime}$ and $\hat{\mathbf{k}}_{\perp i}^{\prime}=\mathbf{k}_{\perp i}^{\prime}-x_{i}^{\prime} \boldsymbol{\Delta}_{\perp} / 2$. For the sake of notation, we henceforth drop the subscripts for the active partons, i.e. for those participating in the subprocess that mediates the photonmeson transition (see Fig. 囵).

The crucial hypothesis in the soft physics approach is now that the soft proton wave functions, i.e. the full wave functions with their perturbative tails removed from them, are dominated by parton virtualities in the range $\left|k_{i}^{2}\right|,\left|k_{i}^{\prime 2}\right| \lesssim \Lambda^{2}$ and by intrinsic transverse parton momenta satisfying $\tilde{\mathbf{k}}_{\perp i}^{2} / x_{i}, \hat{\mathbf{k}}^{\prime 2}{ }_{\perp i} / x_{i}^{\prime} \lesssim \Lambda^{2}$. ${ }^{1}$ With the help of this hypothesis one can show [7] that the subprocess Mandelstam variables, $\hat{s}=(k+q)^{2}$ and $\hat{u}=\left(k^{\prime}-q\right)^{2}$, are respectively equal to $s$ and $u$ up to corrections of order $\Lambda^{2}\left(t \pm Q^{2}\right) / t$ provided $s$ and $-u$ are large on a hadronic scale. This implies that the poles at $\hat{s} \simeq 0$ and $\hat{u} \simeq 0$ appearing in the lowest order Feynman graphs that contribute to the subprocess $\gamma^{*} q \rightarrow M q$ (see Fig. 3) are avoided and, hence, the pole contributions can be neglected. The physical situation is that of a hard parton-level subprocess $\left(\hat{s},-t,-\hat{u} \gg \Lambda^{2}\right)$ and the soft emission and reabsorption of a parton by the proton described by a soft proton matrix element. Hence, we can write the helicity amplitude for the process $\gamma^{*} p \rightarrow M p$ as

$$
\mathcal{M}_{\mu^{\prime} \nu^{\prime}, \mu \nu}^{M(q)}=\sum_{a} e e_{a} B_{a}^{M} \int d^{4} k \theta\left(k^{+}\right) \int \frac{d^{4} z}{(2 \pi)^{4}} e^{i k \cdot z}
$$

\footnotetext{
${ }^{1}$ A restriction to intrinsic transverse momenta $\tilde{\mathbf{k}}_{\perp i}^{2} \lesssim \Lambda^{2}$ instead of $\tilde{\mathbf{k}}_{\perp i}^{2} / x_{i} \lesssim \Lambda^{2}$ fails as is shown in [7]. At least one of the parton virtualities would be of order $\Lambda \sqrt{-t}$ and not $\Lambda^{2}$.
} 

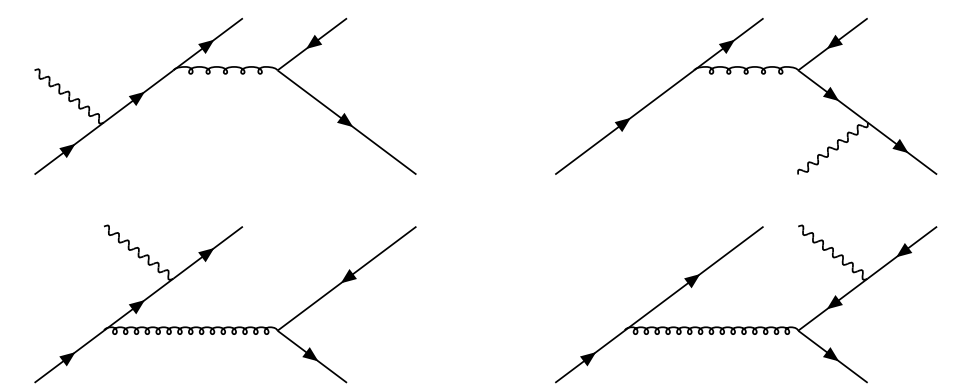

Figure 3: Lowest order Feynman graphs contributing to the subprocess $\gamma^{*} q \rightarrow M q$ where $q$ is either a quark or an antiquark. The upper quark and antiquark lines enter the meson's wave function. The internal curly lines represent hard gluons.

$$
\begin{aligned}
& \times\left[\left\langle p^{\prime} \nu^{\prime}\left|T \bar{\psi}_{a \alpha}(0) \psi_{a \beta}(z)\right| p \nu\right\rangle H_{\mu^{\prime} \mu}^{M(q) \alpha \beta}\left(k^{\prime}, k\right)\right. \\
& \left.+\quad\left\langle p^{\prime} \nu^{\prime}\left|T \bar{\psi}_{a \alpha}(z) \psi_{a \beta}(0)\right| p \nu\right\rangle H_{\mu^{\prime} \mu}^{M(q) \alpha \beta}\left(-k,-k^{\prime}\right)\right],
\end{aligned}
$$

where $H_{\mu^{\prime} \mu}^{M(q)}$ is the tree-level expression for the hard scattering kernel. $\mu$ and $\mu^{\prime}$ respectively denote the helicities of the photon and the meson, $\nu$ and $\nu^{\prime}$ those of the protons. For the sake of legibility we label explicit helicities only by their signs, e.g. we write,+- instead of $+1 / 2,-1 / 2$ for fermions. The helicities are defined in the $\gamma^{*} p$ c.m. frame which is convenient for phenomenological applications and facilitates comparison with other results. On the other hand, the symmetric frame is adapted to discuss the reaction mechanism. The sum runs over quark flavors $a, e_{a}$ being the electric charge of quark $a$ in units of the positron charge $e$ and $B_{a}^{M}$ denotes the meson's flavor wave function. The first term in (5) corresponds to the case where the incoming parton in the subprocess is a quark, the second term corresponds to an incoming antiquark. For the production of flavor neutral vector mesons gluons have to be considered as active partons too. We will discuss this contribution separately below.

Since the subprocess is dominated by a large scale, we can approximate the momenta $k, k^{\prime}$ of the active partons in the subprocess as being on-shell, collinear with their parent hadrons

$$
k \simeq\left[k^{+},-\frac{t}{8 k^{+}},-\frac{1}{2} \Delta_{\perp}\right], \quad k^{\prime} \simeq\left[k^{+},-\frac{t}{8 k^{+}}, \frac{1}{2} \Delta_{\perp}\right] .
$$

The integration over $k^{-}$and $\mathbf{k}_{\perp}$ in (5) can then be performed explicitly leaving an integral $\int d k^{+} \int d z^{-}$and forcing the relative distance of fields in the matrix elements on the light cone, $z \rightarrow \bar{z}=\left[0, z^{-}, \mathbf{0}_{\perp}\right]$. After this the time ordering of the fields can be dropped [23].

The proton matrix element can be viewed as the amplitude for a proton with momentum $p$ emitting the active parton with momentum $k$ and a number of on-shell spectators times the corresponding conjugated amplitude for $p^{\prime}, k^{\prime}$ summed over all spectator configurations, see Fig. 4 . This corresponds to inserting a complete set of intermediate states between quark and antiquark fields in (5). Realizing that at the proton-parton vertices one has large 


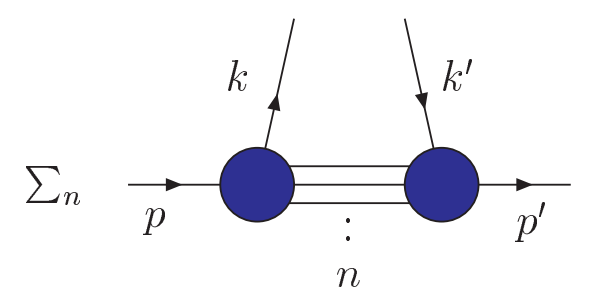

Figure 4: Parton picture of the soft proton matrix element.

plus components but, on account of the central hypothesis of small parton virtualities and small intrinsic transverse momenta, $\tilde{\mathbf{k}}_{\perp i}^{2} / x_{i}, \hat{\mathbf{k}}_{\perp i}^{\prime 2} / x_{i}^{\prime} \lesssim \Lambda^{2}$, one cannot form large kinematical invariants. With this feature of the soft mechanism at hand one can replace the products of fields in (5) by

$$
\begin{aligned}
\bar{\psi}_{\alpha}(0) \psi_{\beta}(\bar{z}) & \rightarrow\left(\frac{1}{2 k^{+}}\right)^{2} \sum_{\lambda, \lambda^{\prime}}\left(\bar{\psi}(0) \gamma^{+} u\left(k^{\prime}, \lambda^{\prime}\right)\right) \\
& \times\left(\bar{u}(k, \lambda) \gamma^{+} \psi(\bar{z})\right) \bar{u}_{\alpha}\left(k^{\prime}, \lambda^{\prime}\right) u_{\beta}(k, \lambda)
\end{aligned}
$$

where $\lambda$ and $\lambda^{\prime}$ denote the helicities of the active partons and $u$ their on-shell spinors. An analogous replacement is possible for the product $\bar{\psi}_{a \alpha}(\bar{z}) \psi_{a \beta}(0)$. In this case antiquark spinors, $v$, appear. Due to this replacement the hard scattering kernels in (5) are multiplied with the spinors for on-shell (anti)quarks

$$
\mathcal{H}_{\mu^{\prime} \lambda^{\prime}, \mu \lambda}^{M(q)}=\bar{u}\left(k^{\prime}, \lambda^{\prime}\right) H_{\mu^{\prime}, \mu}^{M(q)}\left(k^{\prime}, k\right) u(k, \lambda)
$$

which guarantees electromagnetic gauge invariance of our result. The charge conjugation properties of Dirac matrices and spinors relate the subprocess amplitudes involving antiquarks to the quark amplitudes

$$
\bar{v}(k, \lambda) H_{\mu^{\prime} \mu}^{M(q)}\left(-k,-k^{\prime}\right) v\left(k^{\prime}, \lambda^{\prime}\right)=\kappa_{M} \mathcal{H}_{\mu^{\prime} \lambda^{\prime}, \mu \lambda}^{M(q)},
$$

where $\kappa_{V}=-1$ for vector mesons and $\kappa_{P}=+1$ for pseudoscalar ones. The replacement (17) reveals that the plus components of the non-local currents dominate the proton matrix element and that the operators in the matrix elements are in fact the same as those of the leading-twist parton distributions occurring in deep-inelastic lepton-nucleon scattering, DVCS or DVEM. This is a nontrivial dynamical feature of large momentum transfer Compton scattering and electroproduction of mesons, given that, in contrast to the deeply virtual reactions, not only the plus components of the parton momenta but also their minus and transverse components are large now.

As mentioned above we follow the concept used in the calculation of DVEM [5, 6, 24] and treat the formation of the helicity zero mesons $\left(\mu^{\prime}=0\right)$ to leading-twist, lowest order perturbative QCD (cf. Fig. 3). In combination with the disregard of quark masses this 
formation mechanism leads to conservation of quark helicity in the subprocess, $\lambda^{\prime}=\lambda$. This feature and properties of massless spinors allow to simplify the expression (5) further, and to arrive at

$$
\begin{aligned}
\mathcal{M}_{0 \nu^{\prime}, \mu \nu}^{M(q)} & =\frac{1}{4} \sum_{\lambda} \sum_{a} e e_{a} B_{a}^{M} \int \frac{d k^{+}}{k^{+}} \theta\left(k^{+}\right) \int \frac{d z^{-}}{2 \pi} e^{i k^{+} z^{-}} \mathcal{H}_{0 \lambda, \mu \lambda}^{M(q)} \\
& \times\left[\left\langle p^{\prime} \nu^{\prime}\left|\bar{\psi}_{a}(0) \gamma^{+} \psi_{a}(\bar{z})+\kappa_{M} \bar{\psi}_{a}(\bar{z}) \gamma^{+} \psi_{a}(0)\right| p \nu\right\rangle\right. \\
& \left.+\lambda\left\langle p^{\prime} \nu^{\prime}\left|\bar{\psi}_{a}(0) \gamma^{+} \gamma_{5} \psi_{a}(\bar{z})-\kappa_{M} \bar{\psi}_{a}(\bar{z}) \gamma^{+} \gamma_{5} \psi_{a}(0)\right| p \nu\right\rangle\right] .
\end{aligned}
$$

Following [7], we take $k^{+}=p^{+}$, i.e. the light-cone fractions $x=x^{\prime}=1$ in the hard scattering which is in line with the requirement to have no hard parton directly coupling to the protons. Admittedly, the global factor $1 / k^{+}$in $(10)$ cannot be plainly associated with either the hard scattering or the soft matrix element. We therefore choose to keep $k^{+}=x p^{+}$for this factor. We can now pull out the hard scattering amplitude from the integrals and use a form factor decomposition for the integrated proton matrix element [7, 8]

$$
\begin{aligned}
& \int_{0}^{1} \frac{d x}{x} p^{+} \int \frac{d z^{-}}{2 \pi} e^{i x p^{+} z^{-}}\left\langle p^{\prime} \nu^{\prime}\left|\bar{\psi}_{a}(0) \gamma^{+} \psi_{a}(\bar{z})+\kappa_{M} \bar{\psi}_{a}(\bar{z}) \gamma^{+} \psi_{a}(0)\right| p \nu\right\rangle \\
& =R_{V}^{M a}(t) \bar{u}\left(p^{\prime}, \nu^{\prime}\right) \gamma^{+} u(p, \nu)+R_{T}^{M a}(t) \frac{i}{2 m} \bar{u}\left(p^{\prime}, \nu^{\prime}\right) \sigma^{+\beta} \Delta_{\beta} u(p, \nu), \\
& \int_{0}^{1} \frac{d x}{x} p^{+} \int \frac{d z^{-}}{2 \pi} e^{i x p^{+} z^{-}}\left\langle p^{\prime} \nu^{\prime}\left|\bar{\psi}_{a}(0) \gamma^{+} \gamma_{5} \psi_{a}(\bar{z})-\kappa_{M} \bar{\psi}_{a}(\bar{z}) \gamma^{+} \gamma_{5} \psi_{a}(0)\right| p \nu\right\rangle \\
& =R_{A}^{M a}(t) \bar{u}\left(p^{\prime}, \nu^{\prime}\right) \gamma^{+} \gamma_{5} u(p, \nu) .
\end{aligned}
$$

$R_{V}^{M a}, R_{T}^{M a}$ and $R_{A}^{M a}$ are new form factors, depending on the type of the meson, $V$ or $P$, and on the flavor of the active quark. As the definition (11) reveals they are $1 / x$ moments of SPDs at zero skewedness. The link-operator needed to render the definition of the SPDs gauge invariant, is not displayed in (11), i.e. we assume the use of a light-cone gauge combined with an appropriate choice for the integration path which reduces the link operator to unity. Due to time reversal invariance the form factors are real functions. The form factor $R_{T}^{M a}$ is controlled by higher-twist dynamics and is expected to be suppressed by $m^{2} / t$ as compared with $R_{V}^{M a}$ [9]. Since the calculation of the soft contributions is only accurate up to corrections in $\Lambda^{2} / t, R_{T}^{M a}$ is to be omitted for consistency. Hence, we can only calculate the amplitudes conserving the proton helicity. Explicitly they read

$$
\begin{aligned}
\mathcal{M}_{0+, \mu+}^{M(q)}(s, t)= & \frac{e}{2}\left\{\mathcal{H}_{0+, \mu+}^{M(q)}(s, t)\left[R_{V}^{M}(t)+R_{A}^{M}(t)\right]\right. \\
& \left.+\mathcal{H}_{0-, \mu-}^{M(q)}(s, t)\left[R_{V}^{M}(t)-R_{A}^{M}(t)\right]\right\},
\end{aligned}
$$

where the form factors specific to the process $\gamma^{*} p \rightarrow M p$ are defined as

$$
R_{V, A}^{M}(t)=\sum_{a} e_{a} B_{a}^{M} R_{V, A}^{M a}(t)
$$


From parity invariance one has $\mathcal{M}_{0 \nu, \mu \nu}^{M(q)}=-\kappa_{M}(-1)^{\mu} \mathcal{M}_{0-\nu,-\mu-\nu}^{M(q)}$ and an analogous equation for the parton-level amplitudes $\mathcal{H}_{0 \lambda^{\prime}, \mu \lambda}^{M(q)}$. The amplitudes for longitudinally polarized photons simplify as a consequence of parity invariance: the vector form factor, $R_{V}^{M}$, contributes only in the case of vector meson production while the axial vector form factor, $R_{A}^{M}$, contributes in the case of pseudoscalar mesons. This is analogous to DVEM. For transversally polarized photons, on the other hand, both form factors contribute.

Let us now turn to the calculation of the parton-level amplitudes. The mesons are described by their valence Fock components and, for a longitudinally polarized vector meson, we write the corresponding matrix element in the usual way as

$$
\left\langle V, q^{\prime}\left|\bar{\psi}(x) \gamma_{\mu} \psi(y)\right| 0\right\rangle=q_{\mu}^{\prime} f_{V} \int_{0}^{1} d \tau \phi_{V}(\tau) e^{i q^{\prime} \cdot(\tau x+\bar{\tau} y)}
$$

where the proportionality between the meson's polarization vector and its momentum, $q^{\prime}$, for longitudinally polarized vector mesons is employed. For pseudoscalar mesons we have:

$$
\left\langle P, q^{\prime}\left|\bar{\psi}(x) \gamma_{5} \gamma_{\mu} \psi(y)\right| 0\right\rangle=i q_{\mu}^{\prime} f_{P} \int_{0}^{1} d \tau \phi_{P}(\tau) e^{i q^{\prime} \cdot(\tau x+\bar{\tau} y)}
$$

The meson masses are ignored. $\tau$ is the fraction of the meson's momentum the valence quark in the meson carries. The momentum fraction of the antiquark is $\bar{\tau}=1-\tau$. $f_{M}$ is the meson's decay constant and $\phi_{M}$ its distribution amplitude which is normalized as

$$
\int_{0}^{1} d \tau \phi_{M}(\tau)=1
$$

The definitions (14) and (15) are equivalent to the other frequently used ones 10 $\left(\phi^{\prime} / \sqrt{2}\right) f_{V} \phi_{V} /\left(2 \sqrt{2 N_{c}}\right)$ and $\left(\phi^{\prime} \gamma_{5} / \sqrt{2}\right) f_{P} \phi_{P} /\left(2 \sqrt{2 N_{c}}\right)$. The color factor $1 / \sqrt{N_{c}}$ (where $N_{c}$ denotes the number of colors) is not displayed in (14) and (15); it is taken into account in the parton-level amplitudes explicitly.

Working out the Feynman graphs shown in Fig. 3, one finds for the parton-level amplitudes

$$
\mathcal{H}_{0+, \mu+}^{M(q)}=2 \pi \alpha_{s}\left(\mu_{R}\right) f_{M} \frac{C_{F}}{N_{c}} \int_{0}^{1} d \tau \phi_{M}(\tau) f_{\mu}^{(q)}(\tau)
$$

where

$$
\begin{aligned}
f_{+}^{(q)}(\tau) & =\frac{\sqrt{-2 t}}{s+Q^{2}}\left\{\frac{\left(s+Q^{2}\right)\left(\tau s+Q^{2}\right)-\bar{\tau} u Q^{2}}{\bar{\tau} s\left(\tau t-\bar{\tau} Q^{2}\right)}+\frac{\left(s+Q^{2}\right)\left(\tau s-Q^{2}\right)-\bar{\tau} u Q^{2}}{\tau u\left(\bar{\tau} t-\tau Q^{2}\right)}\right\}, \\
f_{-}^{(q)}(\tau) & =-\bar{\tau} \frac{\sqrt{-2 t}}{s+Q^{2}}\left\{\frac{u}{\bar{\tau}\left(\tau t-\bar{\tau} Q^{2}\right)}+\frac{s}{\tau\left(\bar{\tau} t-\tau Q^{2}\right)}\right\}, \\
f_{0}^{(q)}(\tau) & =\frac{2 Q \sqrt{-s u}}{s+Q^{2}}\left\{\frac{u}{s\left(\tau t-\bar{\tau} Q^{2}\right)}+\frac{s+Q^{2}+\bar{\tau} u}{\tau u\left(\bar{\tau} t-\tau Q^{2}\right)}\right\} .
\end{aligned}
$$

$C_{F}=\left(N_{c}^{2}-1\right) /\left(2 N_{c}\right)$ is the usual SU(3) color factor. Parity invariance fixes the amplitudes with negative quark helicities. For the scale of the parton-level amplitudes we choose 
$\mu_{R}=s / 4$ which is roughly the average of the gluon and quark virtualities in the hard process.

In principle the amplitudes (17) hold for all values of $t$ and $Q^{2}$ provided the internal quark and gluon virtualities are sufficiently large. In the limit of either $Q^{2} \rightarrow 0$ or $t \rightarrow 0$ the amplitudes (17) simplify strongly. In the case of photoproduction we find

$$
\begin{aligned}
& \mathcal{H}_{0+,++}^{M(q)}=-2 \pi \alpha_{s}\left(\mu_{R}\right) f_{M} \frac{C_{F}}{N_{c}}\langle 1 / \tau\rangle_{M} \frac{\sqrt{-2 t}}{u}, \\
& \mathcal{H}_{0+,-+}^{M(q)}=2 \pi \alpha_{s}\left(\mu_{R}\right) f_{M} \frac{C_{F}}{N_{c}}\langle 1 / \tau\rangle_{M} \frac{\sqrt{-2 t}}{s},
\end{aligned}
$$

and $\mathcal{H}_{0+, 0+}^{M(q)}=0\left(\propto Q\right.$ for $\left.Q^{2} \rightarrow 0\right)$. In deriving (19) we made use of the symmetry of the distribution amplitude for the mesons of interest under the interchange $\tau \leftrightarrow \bar{\tau}$, $\phi_{M}(\tau)=\phi_{M}(\bar{\tau})$. One observes that only the moment

$$
\langle 1 / \tau\rangle_{M}=\int_{0}^{1} d \tau \frac{\phi_{M}(\tau)}{\tau}
$$

contributes. In the limit of large $Q^{2}$ and small $-t$, the case of DVEM, the amplitude for longitudinally polarized photons, $\mathcal{H}_{0+, 0+}^{M(q)}$, also becomes proportional to the $1 / \tau$ moment, cf. for instance [24], while terms $\propto 1 / \tau^{2}, 1 / \bar{\tau}^{2}$ in the other two amplitudes signal the breakdown of factorization for transversally photons 25]. Inserting the parton-level amplitudes (17) into (12), one obtains the final expressions for the helicity amplitudes.

For flavor-neutral vector meson there is a complication which we now have to discuss, namely gluons have to be considered as active partons as well. Again this situation is similar to DVEM [5, 6, 24]. In the kinematical region of $\Lambda^{2} \ll Q^{2} \ll s$, characteristic of the HERA experiments, the gluon contribution even dominates [26, 27]. We start the calculation of the gluon contribution from an expression similar to (5)

$$
\begin{aligned}
\mathcal{M}^{V(g)} & =\sum_{a} e e_{a} B_{a}^{V} \int d^{4} k \theta\left(k^{+}\right) \int \frac{d^{4} z}{(2 \pi)^{4}} e^{i k \cdot z} \\
& \times\left\langle p^{\prime}\left|T A^{\rho b}(0) A^{\rho^{\prime} b^{\prime}}(z)\right| p\right\rangle H_{\rho \rho^{\prime} b b^{\prime}}^{V(g)}\left(k^{\prime}, k\right),
\end{aligned}
$$

where $k$ and $k^{\prime}$ denote the momenta of the on-shell gluons in the symmetric frame, see (6). $A_{\rho b}$ is the gluon field with color $b$. For the sake of legibility we do not display helicity labels in this equation. The proton matrix element is only non-zero if $b=b^{\prime}$. With this in mind we omit color labels in the following for convenience.

Now, we have to repeat all steps of the derivation of the quark contribution. As there, the use of the approximation (6) forces the relative distance of the fields in the proton matrix elements to the light cone, $z \rightarrow \bar{z}$. The important point is now the use of light-cone gauge, $n \cdot A=0$ (where $n=\left[0,1, \mathbf{0}_{\perp}\right]$ ) which allows to express the gluon field by an integral over the field strength tensor $G_{\nu \mu}[3,5,28]$

$$
A_{\nu}(\bar{z} ; n)=n^{\mu} \int_{0}^{\infty} d \sigma e^{-\varepsilon \sigma} G_{\nu \mu}(\bar{z}+\sigma n) .
$$


(The limit $\varepsilon \rightarrow 0$ is understood.) With the help of arguments similar to those leading to (1]) we can replace the products of fields appearing in (21) by

$$
\begin{aligned}
A^{\rho}(0) A^{\rho^{\prime}}(\bar{z}) & =\sum_{\lambda, \lambda^{\prime}= \pm 1} \epsilon^{\rho}(k, \lambda) \epsilon^{* \rho^{\prime}}\left(k^{\prime}, \lambda^{\prime}\right) \int d \sigma d \sigma^{\prime} e^{-\varepsilon \sigma-\varepsilon^{\prime} \sigma^{\prime}} \\
& \times G_{\mu+}\left(\sigma^{\prime} n\right) G_{\mu^{\prime}+}(\bar{z}+\sigma n) \epsilon^{* \mu}(k, \lambda) \epsilon^{\mu^{\prime}}\left(k^{\prime}, \lambda^{\prime}\right) .
\end{aligned}
$$

In the symmetric frame the polarization vectors of the on-shell gluons read

$$
\epsilon(k, \lambda)=\left[0, \epsilon^{-}, \epsilon_{\perp}(\lambda)\right], \quad \epsilon\left(k^{\prime}, \lambda^{\prime}\right)=\left[0, \epsilon^{\prime-}, \epsilon_{\perp}\left(\lambda^{\prime}\right)\right],
$$

where $\epsilon_{\perp}( \pm 1)=\mp(1, \pm i) / \sqrt{2}$. The minus components need not be specified since they do not contribute in light-cone gauge.

The hard scattering kernels appearing in (21) are contracted by the first set of polarization vectors in (23) which leads to gauge invariant parton-level amplitudes

$$
\mathcal{H}_{0 \lambda^{\prime}, \mu \lambda}^{V(g)}=\epsilon^{* \rho^{\prime}}\left(k^{\prime}, \lambda^{\prime}\right) H_{\rho^{\prime} \rho}^{V(g)}\left(k^{\prime}, k\right) \epsilon^{\rho}(k, \lambda)
$$

Gluon helicity flip $\left(\lambda=-\lambda^{\prime}\right)$ is suppressed in the proton matrix element at large $-t$ since two units of orbital angular momentum are required in order to avoid helicity flips of the proton $\left(\nu=-\nu^{\prime}\right)$. Thus, matrix elements involving helicity flips of the gluons are suppressed at least as $\propto m^{2} / t$ and will be omitted. This argument is of importance only for longitudinally polarized photons because, for $\mu= \pm 1$, the parton-level amplitudes $\mathcal{H}_{0 \lambda, \mu-\lambda}$ come out to zero in any case. For $\lambda=\lambda^{\prime}$ one may decompose the last part of (23) into an unpolarized and a polarized gluon contribution

$$
\begin{aligned}
& G_{\mu+}\left(\sigma^{\prime} n\right) G_{\mu^{\prime}+}(\bar{z}+\sigma n) \epsilon^{* \mu}(k, \lambda) \epsilon^{\mu^{\prime}}\left(k^{\prime}, \lambda\right)= \\
& \frac{1}{2} G_{\mu+}\left(\sigma^{\prime} n\right) G_{\mu^{\prime}+}(\bar{z}+\sigma n)\left[g_{\perp}^{\mu \mu^{\prime}}+\lambda \mathcal{P}^{\mu \mu^{\prime}}\right],
\end{aligned}
$$

where $g_{\perp}^{11}=g_{\perp}^{22}=1$ and $\mathcal{P}^{12}=-\mathcal{P}^{21}=i$ while all other components of these tensors are zero. As for the case of quarks, see (11), we introduce a form factor decomposition for the proton matrix elements of the field strength tensors

$$
\begin{aligned}
\int_{0}^{1} d x p^{+} & \int \frac{d z^{-}}{2 \pi} e^{i x p^{+} z^{-}} \int_{0}^{\infty} d \sigma d \sigma^{\prime} e^{-\varepsilon \sigma-\varepsilon^{\prime} \sigma^{\prime}} \\
& \times\left\langle p^{\prime}, \nu^{\prime}\left|G_{\mu+}\left(\sigma^{\prime} n\right) G_{\mu^{\prime}+}(\bar{z}+\sigma n)\right| p, \nu\right\rangle g_{\perp}^{\mu \mu^{\prime}} \\
& =\frac{\bar{u}\left(p^{\prime}, \nu^{\prime}\right) \gamma_{+} u(p, \nu)}{2 p_{+}} R_{V}^{g}(t)+\frac{i}{2 m} \frac{\bar{u}\left(p^{\prime}, \nu^{\prime}\right) \sigma_{+\nu} \Delta^{\nu} u(p, \nu)}{2 p_{+}} R_{T}^{g}(t) .
\end{aligned}
$$

and

$$
\begin{aligned}
\int_{0}^{1} d x p^{+} & \int \frac{d z^{-}}{2 \pi} e^{i x p^{+} z^{-}} \int_{0}^{\infty} d \sigma d \sigma^{\prime} e^{-\varepsilon \sigma-\varepsilon^{\prime} \sigma^{\prime}} \\
& \times\left\langle p^{\prime}, \nu^{\prime}\left|G_{\mu+}\left(\sigma^{\prime} n\right) G_{\mu^{\prime}+}(\bar{z}+\sigma n)\right| p, \nu\right\rangle \mathcal{P}^{\mu \mu^{\prime}} \\
& =\frac{\bar{u}\left(p^{\prime}, \nu^{\prime}\right) \gamma_{+} \gamma_{5} u(p, \nu)}{2 p_{+}} R_{A}^{g}(t)
\end{aligned}
$$




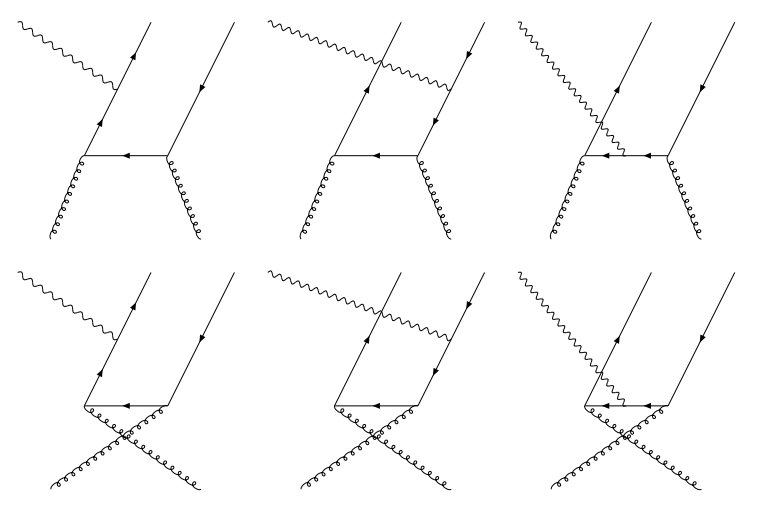

Figure 5: Lowest order Feynman graphs for the subprocess $\gamma^{*} g \rightarrow M g$.

The form factors are related to SPDs at zero skewedness $\left(\mathcal{F}_{\zeta=0}^{g}, \mathcal{K}_{\zeta=0}^{g}\right.$ and $\left.\mathcal{G}_{\zeta=0}^{g}\right)$, e.g.

$$
R_{V}^{g}(t)=\int_{0}^{1} \frac{d x}{x^{2}} \mathcal{F}_{\zeta=0}^{g}(x, t) .
$$

Since the forward limits of $\mathcal{F}_{\zeta}^{g}$ and $\mathcal{G}_{\zeta}^{g}$ are defined in such a way that

$$
x g(x)=\mathcal{F}_{\zeta=0}^{g}(x, t=0), \quad x \Delta g(x)=\mathcal{G}_{\zeta=0}^{g}(x, t=0),
$$

one may still call these form factors $1 / x$-moments of SPDs. Neglecting, as in the case of quarks, $R_{T}^{g}$ we finally arrive at the helicity amplitudes for the gluon contribution

$$
\begin{aligned}
\mathcal{M}_{0+, \mu+}^{V(g)}(s, t) & =\frac{e}{2}\left[\mathcal{H}_{01, \mu 1}^{V(g)}(s, t)\left(R_{V}^{V(g)}(t)+R_{A}^{V(g)}(t)\right)\right. \\
& \left.+\mathcal{H}_{0-1, \mu-1}^{V(g)}(s, t)\left(R_{V}^{V(g)}(t)-R_{A}^{V(g)}(t)\right)\right],
\end{aligned}
$$

where

$$
R_{V, A}^{V g}=\sum_{a} e_{a} B_{a}^{V} R_{V, A}^{g} .
$$

Because of parity invariance $\mathcal{H}_{0-1,-\mu-1}^{V(g)}=(-1)^{\mu} \mathcal{H}_{01, \mu 1}^{V(g)}$ the form factor $R_{A}^{V(g)}$ does not contribute to the amplitudes for longitudinal photons.

The parton-level amplitudes, to be evaluated from the six lowest order Feynman graphs shown in Fig. 5, read

where

$$
\mathcal{H}_{0+, \mu+}^{V(g)}=\frac{2 \pi \alpha_{s}\left(\mu_{R}\right)}{N_{c}} f_{V} \int_{0}^{1} d \tau \phi_{V}(\tau) f_{\mu}^{(g)}(\tau)
$$

$$
\begin{aligned}
f_{+}^{(g)}(\tau) & =\sqrt{\frac{t}{2 s u}} \frac{Q^{2}}{s+Q^{2}} \frac{1}{\tau \bar{\tau}} \frac{t Q^{2}-\left(s+Q^{2}\right)^{2}-4 \tau \bar{\tau} s u}{\left(\bar{\tau} t-\tau Q^{2}\right)\left(\tau t-\bar{\tau} Q^{2}\right)}, \\
f_{-}^{(g)}(\tau) & =\sqrt{\frac{u t}{2 s}} \frac{s}{s+Q^{2}} \frac{1-4 \tau \bar{\tau}}{\tau \bar{\tau}} \frac{Q^{2}}{\left(\bar{\tau} t-\tau Q^{2}\right)\left(\tau t-\bar{\tau} Q^{2}\right)}, \\
f_{0}^{(g)}(\tau) & =\frac{Q}{s+Q^{2}} \frac{1}{\tau \bar{\tau}} \frac{2 \tau \bar{\tau}\left[Q^{2}\left(s+Q^{2}\right)-t\left(s-Q^{2}\right)\right]-t Q^{2}}{\left(\bar{\tau} t-\tau Q^{2}\right)\left(\tau t-\bar{\tau} Q^{2}\right)} .
\end{aligned}
$$


Since the gluon amplitudes only contribute to photoproduction of flavor neutral vector mesons whose associated distribution amplitudes are symmetric under the interchange $\tau \leftrightarrow \bar{\tau}$, we display only the $\tau \leftrightarrow \bar{\tau}$ symmetric part of the amplitudes in (34). The quark amplitudes (18), on the other hand, contribute to the production of flavored mesons too. We, therefore, show the full quark amplitudes although we do not discuss these cases here.

As one may see from (34), the gluon amplitudes vanish in the limit $Q^{2} \rightarrow 0$. Finite $\gamma g \rightarrow V g$ amplitudes may be obtained if meson masses and/or transverse momenta are taken into account (cf. for instance [27, 29]).

The amplitudes (21) have to be added to those given in (12) for vector mesons:

$$
\mathcal{M}_{0 \nu^{\prime}, \mu \nu}^{V}=\mathcal{M}_{0 \nu^{\prime}, \mu \nu}^{V(q)}+\mathcal{M}_{0 \nu^{\prime}, \mu \nu}^{V(g)}
$$

\section{The form factors and the meson distribution am- plitudes}

Before we present numerical results for the observables of electroproduction of mesons, we have to model the new form factors. In Eq. (13) the general composition of these new form factors is presented in terms of the individual flavor contributions. The explicit flavor structure of the form factors for various mesons is given in Tab. 1. In contrast to Compton scattering [0] where the sum runs over all flavors, here the sum is over the valence quarks of the produced mesons. In other words, the meson selects its valence quarks from the proton. The physical situation is thus similar to DVEM in this respect. For vector mesons, also the flavor factors associated with the gluonic form factors (see (31), (32)) are listed in the table.

\begin{tabular}{|c|c|c|}
\hline & $R_{i}^{M}$ & $R_{i}^{V g}$ \\
\hline$\rho^{0}, \pi^{0}$ & $\frac{1}{\sqrt{2}}\left[e_{u} R_{i}^{M u}-e_{d} R_{i}^{M d}\right]$ & $\frac{1}{\sqrt{2}}\left[e_{u}-e_{d}\right] R_{i}^{g}$ \\
\hline$\omega, \eta_{q}$ & $\frac{1}{\sqrt{2}}\left[e_{u} R_{i}^{M u}+e_{d} R_{i}^{M d}\right]$ & $\frac{1}{\sqrt{2}}\left[e_{u}+e_{d}\right] R_{i}^{g}$ \\
\hline$\phi, \eta_{s}$ & $e_{s} R_{i}^{M s}$ & $e_{s} R_{i}^{g}$ \\
\hline
\end{tabular}

Table 1: Flavor composition of the form factors $R_{i}^{M}(i=V, A)$ for pseudoscalar and vector mesons and the flavor factors for the gluonic form factors.

$\omega-\phi$ mixing is ignored since the corresponding mixing angle is very small. $\eta-\eta^{\prime}$ mixing, on the other hand, is taken into account. Following [30], we work in the quark flavor basis and write

$$
\begin{aligned}
\eta & =\cos \phi_{P} \eta_{q}-\sin \phi_{P} \eta_{s} \\
\eta^{\prime} & =\sin \phi_{P} \eta_{q}+\cos \phi_{P} \eta_{s} .
\end{aligned}
$$


$\eta_{q}$ is a state built from $u$ and $d$ quarks only while $\eta_{s}$ is a $s \bar{s}$ state. The parameters of that $\eta-\eta^{\prime}$ mixing scheme, the mixing angle, $\phi_{P}$, and the decay constants, $f_{q}$ and $f_{s}$, of the basis states are determined in [30] on exploiting the divergencies of the axial vector currents which embody the axial vector anomaly. For the mixing angle a value of $39.2^{\circ}$ is found in [30].

As an inspection of Eqs. (10) and (11) reveals the form factors $R_{V, A}^{V a}$ are exactly the same as those appearing in Compton scattering [0, 8]. Thus, in principle, from a combined analysis of data on Compton scattering and production cross section for various mesons, one may extract information on the form factors for individual flavors from experiment. This allows to test the soft mechanism independent of a specific model for the form factors.

For a numerical estimate of the form factors we use the model proposed in Ref. [7]. In a frame where $\Delta^{+}=0$ the SPDs and, hence, the form factors can be represented as overlaps of light-cone wave functions summed over all Fock states in close analogy to the familiar Drell-Yan formula [31]. A detailed discussion of that overlap representation is given in Refs. [7, 32]. Each $N$-particle Fock state is described by a number of terms, each with its own momentum space wave function $\Psi_{N \beta}$, where $\beta$ labels different spin-flavor combinations of the $N$ partons. Assuming a single Gaussian $\tilde{k}_{i \perp}$-dependence of the soft Fock state wave functions

$$
\Psi_{N \beta}\left(x_{i}, \tilde{k}_{\perp i}\right) \propto \exp \left[-a_{N}^{2} \sum_{i=1}^{N} \frac{\tilde{k}_{\perp i}^{2}}{x_{i}}\right]
$$

one can explicitly carry out the momentum integration in the overlap formula. The ansatz (37) satisfies various theoretical requirements [33, 34] and is in line with our central hypothesis that the soft hadronic wave functions are dominated by transverse momenta with $\tilde{k}_{\perp i}^{2} / x_{i} \leq \Lambda^{2}$, necessary to achieve the factorization of the electroproduction amplitudes into soft and hard parts ${ }^{2}$. The results of the transverse momentum integration for the vector and axial vector form factors are respectively related with the Fock state contributions to the unpolarized $\left(q_{a}(x), g(x)\right)$ and polarized $\left(\Delta q_{a}(x), \Delta g(x)\right)$ parton distributions. For simplicity one may assume a common transverse size parameter $a_{N}=\hat{a}$ for all Fock states which seems to be a reasonable approximation since, for large - $t$, the main contribution to the overlap integral is only due to a limited number of Fock states [7]. This simplification immediately allows one to sum over the Fock states without specifying the $x_{i}$-dependence of the wave functions. One then arrives at the following model for the form factors for individual flavors $(a=u, d, s)$ :

$$
\begin{aligned}
& R_{V}^{V a}(t)=\int_{0}^{1} \frac{d x}{x} \exp \left[\frac{1}{2} \hat{a}^{2} t \frac{1-x}{x}\right]\left\{q_{a}(x)+\bar{q}_{a}(x)\right\} \\
& R_{V}^{P a}(t)=\int_{0}^{1} \frac{d x}{x} \exp \left[\frac{1}{2} \hat{a}^{2} t \frac{1-x}{x}\right]\left\{q_{a}(x)-\bar{q}_{a}(x)\right\},
\end{aligned}
$$

\footnotetext{
${ }^{2}$ The wave function (37), perhaps multiplied by a polynomial in the $x_{i}$, is not continuous in the endpoints $x_{i}=\tilde{k}_{\perp i}=0(i=1,2$ or 3$)$. It can, however, be shown [32 that the overlaps evaluated from such wave functions, are infrared stable, i.e. they are not dominated by contributions from regions of very small $x_{i}$ and $\tilde{k}_{\perp i}$.
} 


$$
R_{V}^{g}(t)=\int_{0}^{1} \frac{d x}{x} \exp \left[\frac{1}{2} \hat{a}^{2} t \frac{1-x}{x}\right] g(x) .
$$

The corresponding axial vector form factors are obtained from (38) by replacing the unpolarized parton distributions $q_{a}, g$ with the polarized ones, $\Delta q_{a}, \Delta g$.

As shown in [7] an evaluation of these form factors from the parton distributions of Glück et al. (GRV) [35] (taken at a scale of $1 \mathrm{GeV}$ ) and with $\hat{a} \simeq 1 \mathrm{GeV}^{-1}$, leads to results for Compton scattering in fair agreement with experiment. In order to improve the model (38) the lowest three Fock states were modeled explicitly in [0] assuming specific distribution amplitudes, e.g.

$$
\phi_{123}\left(x_{i}\right)=60 x_{1} x_{2} x_{3}\left(1+3 x_{1}\right),
$$

for the valence Fock state [16]. The form factors (38) for the quarks are then evaluated from these three lowest Fock states (with $a_{3}=a_{4}=a_{5}=0.75 \mathrm{GeV}^{-1}$ ) and the contribution from all higher Fock states is estimated by setting $\left(a_{N}=1.3 a_{3}\right.$ for $\left.N>5\right)$

$$
\sum_{N>5} q_{a}^{(N)}(x)=q_{a}(x)-\sum_{N=3,4,5} q_{a}^{(N)}(x) .
$$

The $q_{a}(x)$ are taken from the GRV parameterization [35] and the three lowest Fock state contribution $q_{a}^{(N=3,4,5)}(x)$ are evaluated from the light-cone wave functions. This model provides a good fit to Compton scattering and to the proton form factor $F_{1}$ (by expressions similar to (38) [7]). In Fig. 6 numerical results for the vector and axial vector form factors are shown. The simplifying model assumption of unpolarized gluons and sea quarks has the consequence of a zero gluon form factor $R_{A}^{g}$. Most of the strange form factors are therefore zero too:

$$
R_{V}^{P s}=R_{A}^{V s}=R_{A}^{P s}=0 .
$$

Only $R_{V}^{V s}$ is non-zero, even though very small. The form factors for $u$ quarks are largest. They approximately behave as $1 / t^{2}$ in the momentum transfer region from about 5 to $15 \mathrm{GeV}^{2}$; for the other flavors and for the gluon that range is shifted to somewhat smaller values of $-t$. With increasing $-t$ the form factors for $u$ and $d$ quarks gradually turn into the soft physics asymptotic $\propto 1 / t^{4}$, while the other form factors decrease faster. The leading powers of $1 / t$ in the asymptotic behavior of the form factors (38) follow from the $x_{i}$-dependence of the model wave functions at the end points (see for instance Eq. (39)) [7]. In the region where the form factors drop as $1 / t^{4}$ or faster, which is above $100 \mathrm{GeV}^{2}$, the perturbative contribution will take the lead.

The other soft physics information required in our approach is that of the form of the meson's distribution amplitude. From analyzes of the pion-photon transition form factor (see for instance [12, 13, 14]) it became evident that the pion's distribution amplitude (its formal definition is given in (15)) is close to the asymptotic form

$$
\phi_{\mathrm{AS}}(\tau)=6 \tau(1-\tau) .
$$

This result is supported by the instanton model [36] and by recent QCD sum rule studies [37]. The analyzes of the $\eta$ - and $\eta^{\prime}$-photon transition form factors revealed that the $\eta_{q}$ 

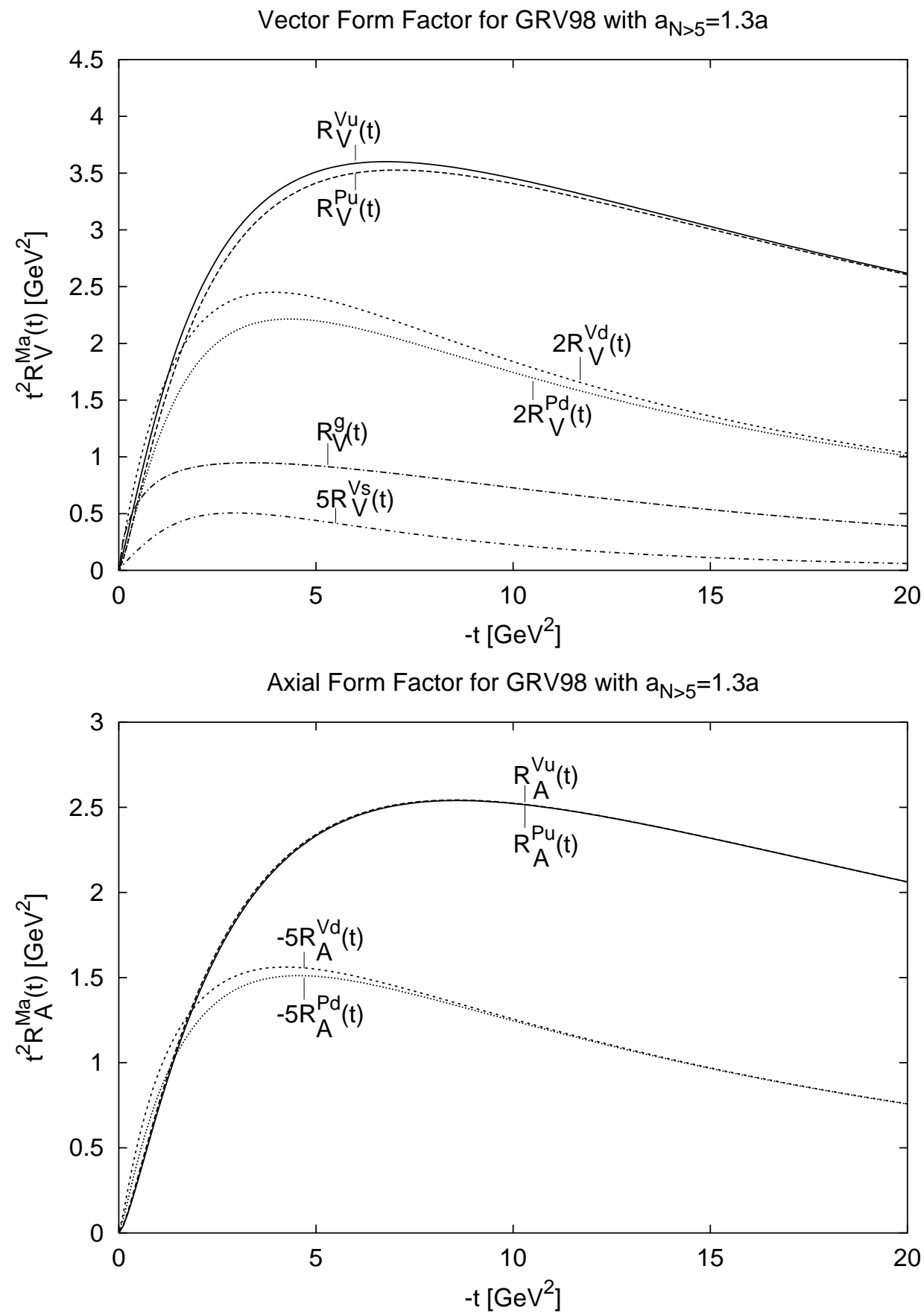

Figure 6: The form factors $R_{V}^{M a}, R_{A}^{M a}$ and $R_{V}^{g}$, scaled by $t^{2}$, as evaluated from the overlap model proposed in [0]. 
distribution amplitude is close to the form (42), too [12, 38]. Although the transition form factor data are compatible with the asymptotic distribution amplitude for the $\eta_{s}$ as well, a somewhat narrower one cannot be excluded. For vector mesons no phenomenological information is available but QCD sum rules [39] taught us that the distribution amplitudes for longitudinally polarized vector mesons can also be approximated by (42). In order to keep matters simple we therefore choose the form (42) for all mesons. We expect that the uncertainties in the predicted production cross sections due this choice do not exceed $10-15 \%$. Associated with the distribution amplitude (42) is a value of 3 for the $1 / \tau$ moment (20).

For the meson decay constants we use the values 40

$$
\begin{aligned}
& f_{\pi}=132 \mathrm{MeV}, \quad f_{\rho}=216 \mathrm{MeV} \\
& f_{\omega}=195 \mathrm{MeV}, \quad f_{\phi}=237 \mathrm{MeV}
\end{aligned}
$$

and for the decay constants of the states $\eta_{q}$ and $\eta_{s}$ 30

$$
f_{q}=141 \mathrm{MeV}, \quad f_{s}=177 \mathrm{MeV} .
$$

\section{Photoproduction of mesons}

Using (12) and (19), we obtain for the photoproduction cross section of pseudoscalar mesons

$$
\begin{aligned}
\frac{d \sigma^{P}}{d t} & =\frac{1}{2} \alpha_{\mathrm{em}}\left[\pi \alpha_{s}\left(\mu_{R}\right) f_{P}\langle 1 / \tau\rangle_{P} \frac{C_{F}}{N_{C}}\right]^{2} \\
& \times \frac{-t}{u^{2} s^{4}}\left\{(s-u)^{2}\left(R_{V}^{P}(t)\right)^{2}+t^{2}\left(R_{A}^{P}(t)\right)^{2}\right\} .
\end{aligned}
$$

The corresponding expressions for photoproduction of vector mesons are a bit more complicated due to the occurrence of the gluonic contribution. In Fig. I we show, as an typical example, the soft physics contribution to the large momentum transfer photoproduction cross section of uncharged pions as evaluated from the form factors discussed in Sect. 3. We see that at fixed scattering angle the cross section approximately exhibits the $s^{7}$-scaling as predicted by dimensional counting [41]. This scaling behavior holds in the soft physics approach as long as the form factors $R_{V, A}^{M}$ behave as $1 / t^{2}$ (see Fig. 6). Similar results are found for the production of the other mesons.

As compared to experiment (at $s \simeq 10 \mathrm{GeV}^{2}$ ) [20, 22] the soft physics contributions are too small by orders of magnitude. This can easily be understood by evaluating the ratio of $\pi^{0}$ production and Compton cross section [7]

$$
\frac{d \sigma\left(\gamma p \rightarrow \pi^{0} p\right)}{d \sigma(\gamma p \rightarrow \gamma p)}=\frac{-t}{s} \frac{\alpha_{s}^{2}\left(\mu_{R}\right)}{\alpha_{\mathrm{em}}} \frac{f_{\pi}^{2}<1 / \tau>_{\pi}^{2}}{s} c_{s o f t}
$$

where $c_{\text {soft }}$, a ratio of form factors and kinematical factors, is of order 1 . The ratio of the two cross sections is therefore about $2 \mathrm{GeV}^{2} / s$, i.e. much smaller than unity in contradiction 


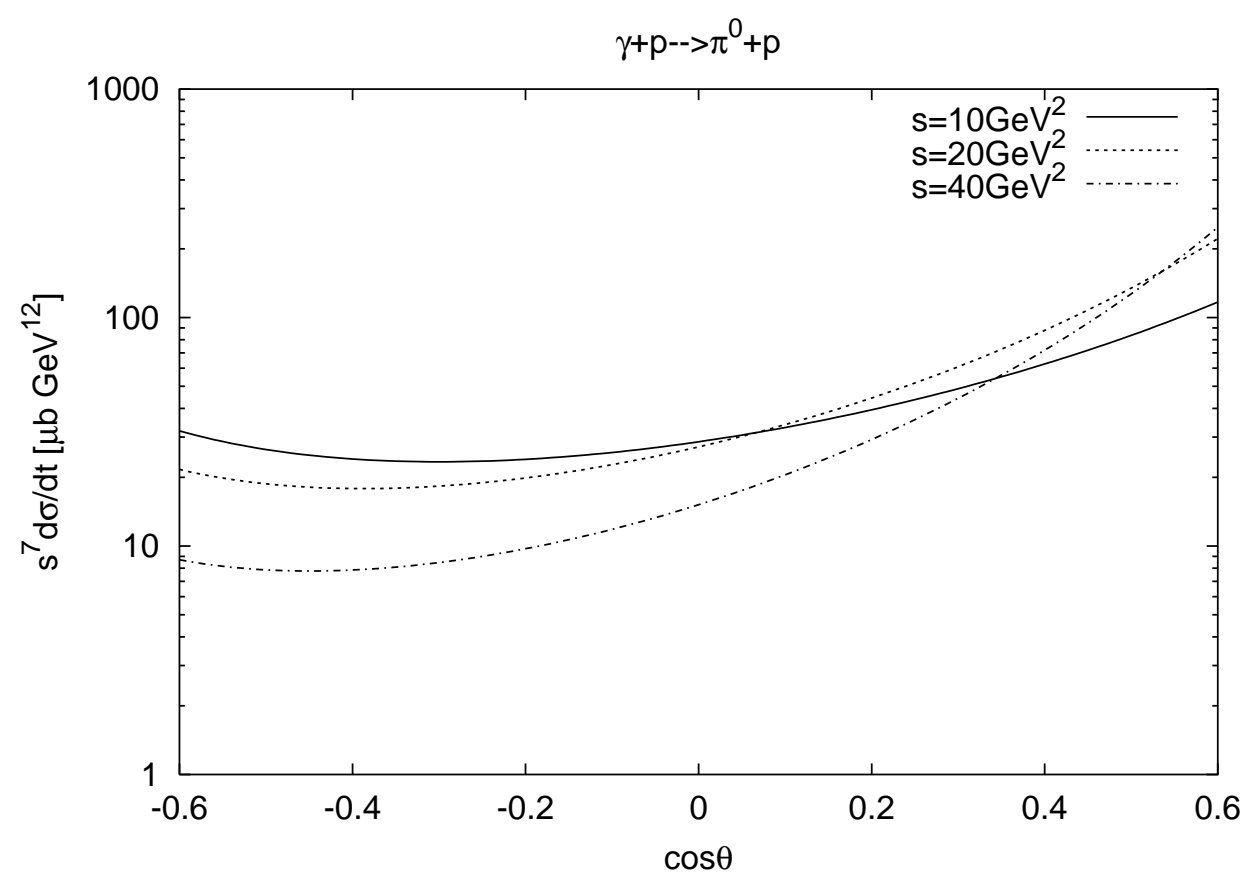

Figure 7: The soft physics contribution to the cross section for photoproduction of $\pi^{0}$ scaled by $s^{7}$ versus $\cos \theta$, where $\theta$ is the scattering angle in the $\gamma^{*} p$ c.m. system.

to experiment [20], where the ratio is about 50 (at $s \simeq 10 \mathrm{GeV}^{2}$, and a $\gamma^{*} p$ c.m. scattering angle, $\theta$, of $90^{\circ}$ ). Mainly responsible for the small ratio (46) is the perturbative formation of the meson which only probes small quark-antiquark separations in the meson. The amplitude is, therefore, proportional to the meson's decay constant which, for dimensional reasons, is to be scaled by $\sqrt{s}$. The $\langle 1 / \tau\rangle_{\pi}$ moment, appearing as a consequence of the perturbative meson formation, cannot compensate the small ratio $f_{\pi} / \sqrt{s}$.

The ratio (46) also holds in perturbative calculations, in the pure quark picture [21] as well as in the diquark model, a variant of the standard perturbative approach in which diquarks are considered as quasi-elementary constituents of baryons [42]. The factor $c_{\text {pert }}$ may, however, be larger than unity. Although there is no obvious enhancement in any of the many Feynman graphs contributing to the perturbative amplitude, the graphs may conspire in such a way that a large value of $c_{\text {pert }}$ is built up. In order to see whether or not this is the case, an explicit and reliable calculation of meson production within the perturbative approach is called for.

The observation of a relatively large photoproduction cross section in experiment is in line with the power law behavior in $s$ at fixed scattering angle. $s^{-n}$-fits to the present data in the range $6.5 \mathrm{GeV}^{2} \lesssim s \lesssim 12 \mathrm{GeV}^{2}$ and $50^{\circ}<\theta<130^{\circ}$ provide [20]:

$$
\begin{array}{ll}
\gamma p \rightarrow \pi^{0} p: & n=8.0 \pm 0.1 \\
\gamma p \rightarrow \gamma p: & n=6.1 \pm 0.3 .
\end{array}
$$


For the $\gamma p \rightarrow\left(\rho^{0}+\omega\right) p$ data [22] the statistics does not allow a meaningful determination of the power $n$. However, $n$ seems to be larger than 7 , rather compatible with 8 . For Compton scattering the power is compatible with dimensional counting, while for $\pi^{0}$ production, and possibly for the sum of $\rho^{0}$ and $\omega$ production, the value of $n$ rather equals that one observed in elastic $\pi p$ scattering [43] ( $n \simeq 8$; data are averaged over resonance-like structures) ? . Admittedly, the photoproduction data are rather poor and need confirmation. Data on photoproduction of $\phi$ mesons will become available from the TJlab soon 44 which will perhaps allow a determination ot the power $n$ for that reaction.

Both the observations in the large momentum transfer photoproduction data, the large powers of $s$ at fixed scattering angle and the large cross sections, indicates that another dynamical mechanism is at work here. It is tempting to assign it to the hadronic component of the photon. This proposition is supported by a vector meson dominance (VMD) estimate of the photoproduction cross section. Combined with quark model ideas VMD, for instance, relates photoproduction of $\rho^{0}$-mesons to elastic pion-nucleon scattering 45

$$
\frac{d \sigma}{d t}\left(\gamma p \rightarrow \rho^{0} p\right)=\alpha_{\mathrm{em}} \frac{\pi f_{\rho}^{2}}{m_{\rho}^{2}}\left[\frac{d \sigma}{d t}\left(\pi^{+} p \rightarrow \pi^{+} p\right)+\frac{d \sigma}{d t}\left(\pi^{-} p \rightarrow \pi^{-} p\right)\right]
$$

This relation is satisfied by experiment within a factor of 2-3 [22]. With respect to the uncertainties arising from possible spin effects and the poor quality of data this may be considered as fair agreement. Thus, it seems that photoproduction of $\rho^{0}$ and $\pi^{0}-$ and likely of other mesons - is indeed controlled by the hadronic component of the photon. In this case one would expect the produced vector mesons to be polarized transversally rather than longitudinally. Since the fixed-angle energy dependencies of the contributions from the hadronic component of the photon $\left(\simeq s^{-8}\right)$ and from the soft mechanism $\left(\simeq s^{-7}\right)$ are so close, much higher energies are needed before the soft contribution (and/or the perturbative one) will control photoproduction of mesons. We, therefore, refrain from presenting more predictions from the soft physics approach for photoproduction of mesons. We stress that our approach to photoproduction requires high energies, large momentum transfer and small values of $|\cos \theta|$. If $s /-t \gg 1$ Pomeron exchange becomes dominant, see for instance [46].

One may wonder whether Compton scattering is also dominated by the hadronic component of the photon. However, the analogous VMD estimate, with both the photons replaced by vector mesons, provides values for the Compton cross section that are about an order of magnitude below experiment 20] at $s \simeq 10 \mathrm{GeV}^{2}$. Moreover, the Compton cross section exhibits $s^{6}$-scaling and not an $s^{8}$ one as would be the case if the hadronic component of the photon dominates. Thus, the simplest elementary process, elastic scattering of point-like photons from quarks, dominates Compton scattering off protons already at rather low energies 47.

${ }^{3}$ We recall that a $s^{8}$-scaling of the fixed-angle meson baryon cross section is easily accounted for by the soft physics approach in the relevant region of energy [9]. 


\section{$5 \quad$ Electroproduction of mesons}

Let us now discuss our results for large momentum transfer electroproduction. As is wellknown the cross section for $e p \rightarrow e p M$ can be decomposed as follows

$$
\begin{aligned}
\frac{d^{4} \sigma^{M}}{d s d Q^{2} d t d \varphi} & =\frac{\alpha_{\mathrm{em}} s}{16 \pi^{2} E_{L}^{2} m^{2} Q^{2}(1-\epsilon)} \\
& \times\left(\frac{d \sigma_{T}^{M}}{d t}+\epsilon \frac{d \sigma_{L}^{M}}{d t}+2 \epsilon \cos 2 \varphi \frac{d \sigma_{T T}^{M}}{d t}+\sqrt{2 \epsilon(1+\epsilon)} \cos \varphi \frac{d \sigma_{L T}^{M}}{d t}\right)
\end{aligned}
$$

where $\varphi$ denotes the azimuthal angle between the hadronic and leptonic scattering planes. $E_{L}$ is the energy of the incoming electron in the laboratory frame and $\epsilon$ is the ratio of longitudinal to transverse photon flux. Details of the kinematics can be found for instance in Ref. [48]. The partial cross sections in (48) read:

(i) The cross sections for transverse photons (reducing to the unpolarized cross section for photoproduction of mesons, i.e. for $Q^{2}=0$ ) and for longitudinal photons,

$$
\begin{aligned}
& \frac{d \sigma_{T}^{M}}{d t}=\frac{1}{32 \pi s\left(s+Q^{2}\right)} \sum_{\nu^{\prime}, \nu}\left|\mathcal{M}_{0 \nu^{\prime},+\nu}^{M}\right|^{2}, \\
& \frac{d \sigma_{L}^{M}}{d t}=\frac{1}{32 \pi s\left(s+Q^{2}\right)} \sum_{\nu^{\prime}, \nu}\left|\mathcal{M}_{0 \nu^{\prime}, 0 \nu}^{M}\right|^{2} .
\end{aligned}
$$

(ii) The transverse-transverse and longitudinal-transverse interference terms

$$
\begin{aligned}
\frac{d \sigma_{T T}^{M}}{d t} & =-\frac{1}{64 \pi s\left(s+Q^{2}\right)} \operatorname{Re} \sum_{\nu^{\prime}, \nu} \mathcal{M}_{0 \nu^{\prime},+\nu}^{M *} \mathcal{M}_{0 \nu^{\prime},-\nu}^{M}, \\
\frac{d \sigma_{L T}^{M}}{d t} & =-\frac{\sqrt{2}}{64 \pi s\left(s+Q^{2}\right)} \operatorname{Re} \sum_{\nu^{\prime}, \nu} \mathcal{M}_{0 \nu^{\prime}, 0 \nu}^{M *}\left[\mathcal{M}_{0 \nu^{\prime},+\nu}^{M}-\mathcal{M}_{0 \nu^{\prime},-\nu}^{M}\right] .
\end{aligned}
$$

In Fig. 8 we show, as a typical example, the $\rho^{0}$ production cross section for longitudinally and transversally polarized photon as a function of $Q^{2}$ at a scattering angle of $90^{\circ}$. Except for small $Q^{2}\left(Q^{2} / s<<1\right)$ the longitudinal cross section

$$
\begin{aligned}
\frac{d \sigma_{L}^{M}}{d t} & =\frac{\alpha_{\mathrm{em}}}{4 N_{c}^{2}} \frac{\left[\pi \alpha_{s}\left(\mu_{R}\right) f_{M} C_{F}\right]^{2}}{s\left(s+Q^{2}\right)}\left\{\int_{0}^{1} d \tau \phi_{M}(\tau)\right. \\
& \times\left[\left(1-\kappa_{M}\right)\left[f_{0}^{(q)}(\tau) R_{V}^{M}(t)+\frac{1}{C_{F}} f_{0}^{(g)}(\tau) R_{V}^{M g}(t)\right]\right. \\
& \left.\left.+\left(1+\kappa_{M}\right) f_{0}^{(q)}(\tau) R_{A}^{M}(t)\right]\right\}^{2}
\end{aligned}
$$

dominates, i.e. the cross section that conserves $s$-channel helicity. Again we have similarity to DVEM. Also similar is the fact that the longitudinal cross section for the production of vector mesons is associated with the vector form factors, $R_{V}^{V}$, while in the case of 


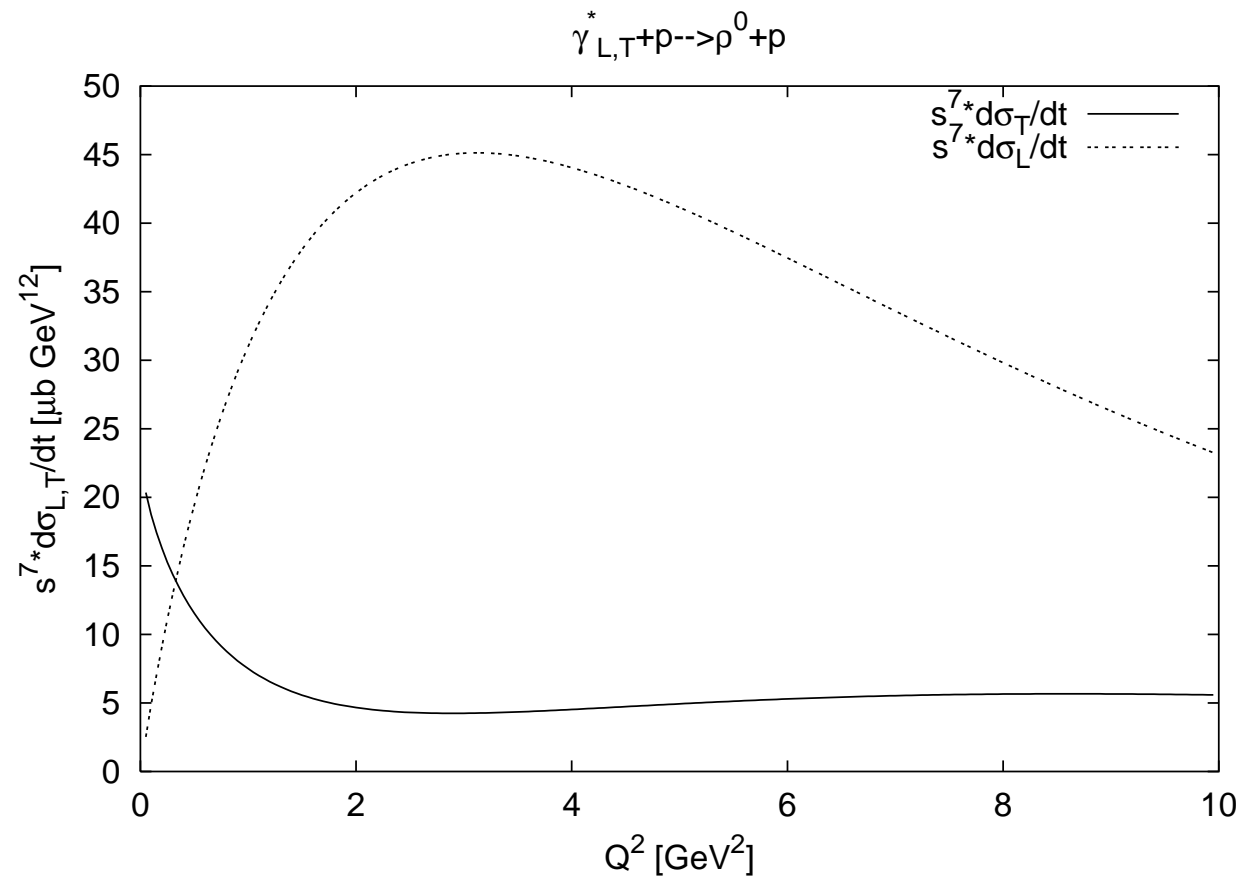

Figure 8: The transverse and longitudinal cross sections for the photoproduction of $\rho^{0}$ mesons at $s=40 \mathrm{GeV}^{2}$ and $\theta=90^{\circ}$.

pseudoscalar mesons it is connected with the axial vector ones, $R_{A}^{P}$. In contrast to the limiting cases of either $t \rightarrow 0$ or $Q^{2} \rightarrow 0$ the explicit form of the mesons distribution amplitude is required in the evaluation of the large momentum transfer electroproduction cross section. Tuning the ratio $Q^{2} / t$ details of the distribution amplitude can be explored. Even if the form factors behave $\propto 1 / t^{2}$ strictly, $d \sigma / d t$ does not exhibit $s^{7}$-scaling. Despite of this we keep multiplying the cross section by $s^{7}$ since this compensates most of the energy dependence.

The hadronic component of the photon vanishes with increasing $Q^{2}$ rapidly, approximately as $m_{V}^{2} /\left(Q^{2}+m_{V}^{2}\right)$ in the amplitude. This is for instance, clearly visible in the integrated cross section for $\rho^{0}$ production: while, at low $Q^{2}\left(\lesssim 2 \mathrm{GeV}^{2}\right)$, its energy dependence is very similar to that of total cross sections for elastic hadron-hadron scattering, it is much steeper at large $Q^{2} 449$. The steep rise of the $\rho^{0}$ cross section is correlated with the behaviour of the gluon SPD for $0<x^{\prime} \leq x \ll 1$ which should reflect the strong increase of the gluon distribution for $x \rightarrow 0$ [5, 27]. By virtue of the rapidly decreasing hadronic component of the photon and the strong rise of the longitudinal cross section (see Fig. 8) with increasing $Q^{2}$, we expect the soft physics approach to be applicable for photon virtualities larger than about $2-3 \mathrm{GeV}^{2}$ provided $s,-t$ and $-u$ are large.

Results for the partial cross sections for electroproduction of $\rho^{0}$-mesons are shown in Fig. 9 (at $s=20 \mathrm{GeV}^{2}$ and $40 \mathrm{GeV}^{2}$ ). We see that the longitudinal cross section is dominant in the region of small $|\cos \theta|$. For larger values of $|\cos \theta|$ the transverse cross 

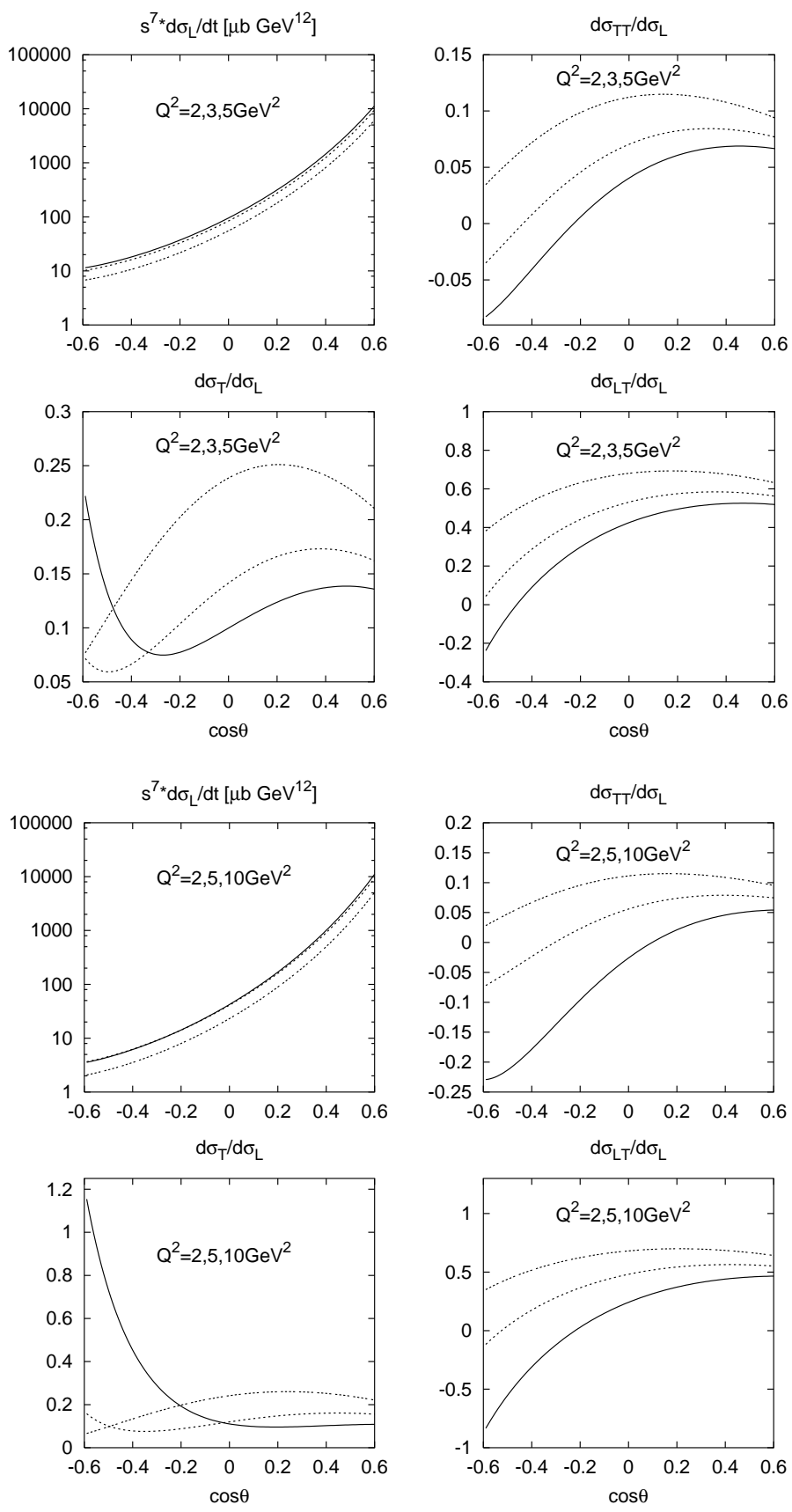

Figure 9: The partial cross sections for electroproduction of $\rho^{0}$ mesons versus $\cos \theta$ at $s=20 \mathrm{GeV}^{2}$ and $Q^{2}=2,3,5 \mathrm{GeV}^{2}$ (top) and at $s=40 \mathrm{GeV}^{2}, Q^{2}=2,5,10 \mathrm{GeV}^{2}$ (bottom), plotted as solid, dotted and dashed lines, respectively. 


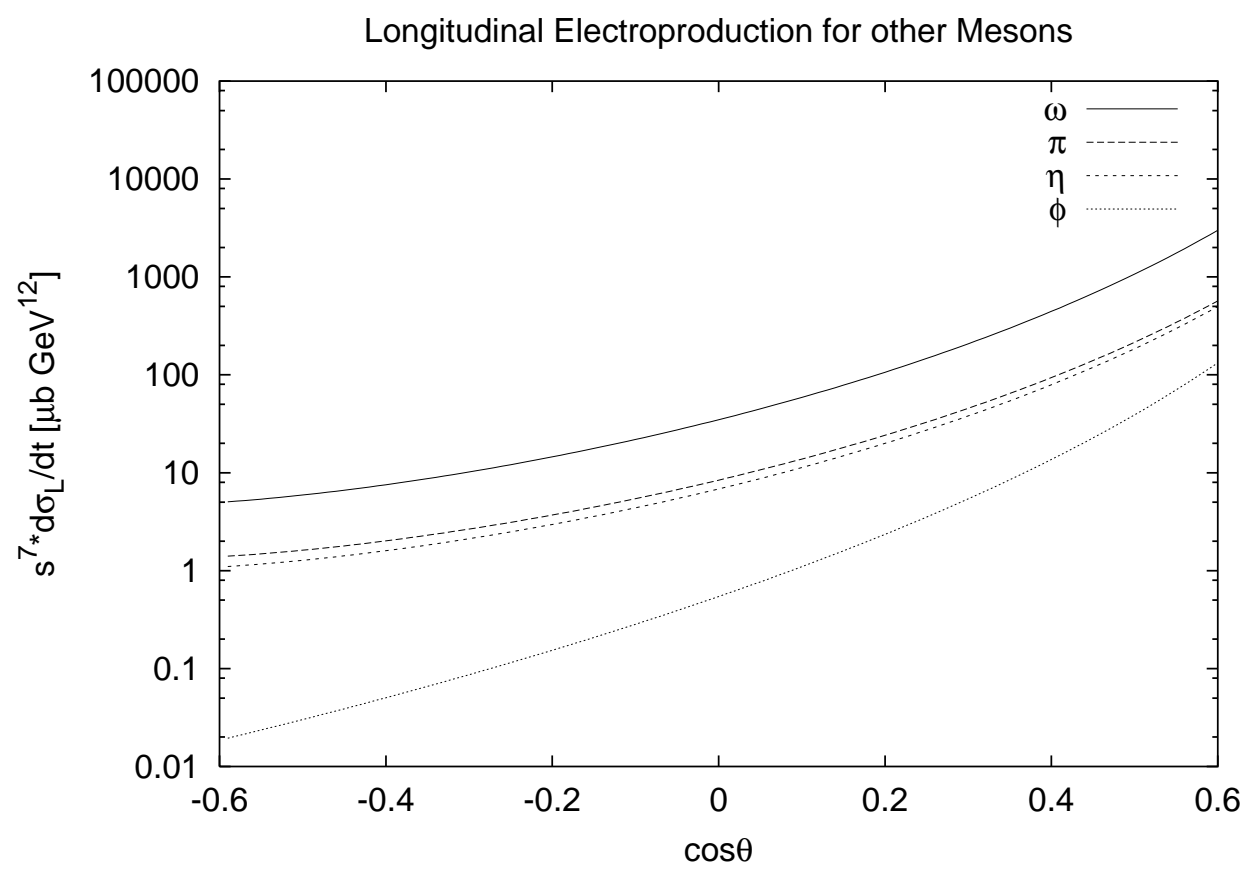

Figure 10: The longitudinal cross section, scaled by $s^{7}$, for electroproduction of $\pi^{0}, \eta, \omega$ and $\phi$ mesons versus $\cos \theta$ at $s=20 \mathrm{GeV}^{2}$ and $Q^{2}=3 \mathrm{GeV}^{2}$.

section as well as the longitudinal-transverse interference become sizeable.

In order to demonstrate the relative magnitude of the production cross sections for various mesons we display predictions for the scaled longitudinal cross sections at $s=$ $20 \mathrm{GeV}^{2}$ and $Q^{2}=3 \mathrm{GeV}^{2}$ in Fig. 10. The gluonic contributions to the cross sections for vector mesons are generally small in the kinematical region of interest. This is obvious from the relative strength of the quark and gluon form factors, see Fig. 6. The exceptional case is the $\phi$ meson. The only quark form factor contributing, $R_{V}^{V s}$, is very small and the gluonic contribution therefore dominates the production of $\phi$ mesons. Since the form factor $R_{A}^{P s}$ is zero in the model (see Eq. (41)) the ratio of longitudinal cross sections for the production of $\eta^{\prime}$ and $\eta$ mesons is given by the square of the tangent of the pseudoscalar meson mixing angle, see (36).

Finally we comment on the accuracy of our calculation. As we said repeatedly, large momentum transfer and photon virtualities larger than about $2-3 \mathrm{GeV}^{2}$ are required for the dominance of the soft mechanism. However, the momentum transfer should not be too large since then the pure perturbative contribution [10, 21] will become important. The onset of the perturbative regime is expected to be beyond $t \simeq-100 \mathrm{GeV}^{2}$ [7]. Even in the soft regime one has to be aware of corrections. For instance, the lowest-order, leading twist perturbative formation of the mesons may be subject to substantial corrections of perturbative and/or soft origin. These may give rise to a $\kappa$-factor in the normalization of the electroproduction cross section as is known from the Drell-Yan process and, according 
to Martin et al. [27], is required in DVEM at least at small $Q^{2} / s$.

\section{Summary}

In the present work electroproduction of flavor neutral pseudoscalar and longitudinally polarized vector mesons at large $s,-t$ and $-u$ is investigated. The photon virtuality is not considered as a large scale; therefore the limit $Q^{2} \rightarrow 0$ is included in the investigation. This study of electroproduction is complementary to the case of DVEM where $Q^{2}$ is large and $-t$ small. Based on the central assumption of the dominance of small parton virtualities and small intrinsic transverse momenta in the proton's light-cone wave function we have shown that, like in Compton scattering [7], the electroproduction amplitudes factorize in hard parton-level subprocess amplitudes and soft proton matrix elements described by the same type of form factors as appear in Compton scattering. These form factors represent $1 / x$-moments of SPDs. The soft mechanism bears resemblance to the dynamics controlling DVEM [5, 6, 24, 26, 27] in many respects: The same parton-level subprocesses occur, the longitudinal cross section dominates (if $|\cos \theta|$ is small and $Q^{2}$ not too small) and the soft information on the proton is encoded in SPDs. Different is that, in the large momentum transfer region, a symmetric frame with zero skewedness can be chosen which entails the formation of $1 / x$-moments of the SPDs, i.e. the appearance of new form factors. For asymptotically large momentum transfer the perturbative contribution [10] will take the lead, the soft contribution, discussed here, then presents a power correction to it. We emphasize that the dimensional counting rule behaviour, i.e. $s^{7}$-scaling, approximately holds for photoproduction of mesons in the soft physics approach for a limited range of energy.

The new form factors, characteristic of large momentum transfer Compton scattering and electroproduction of mesons, can in principle be extracted from experiment by Rosenbluth-type separations [50]. Their measurements would provide information on the large momentum transfer behavior of the proton SPDs and would allow to test models for them. Moreover, the experimental verification of their energy independence would constitute a severe test of the soft physics approach.

Based on a light-cone wave function overlap model for the form factors we have presented detailed predictions for electroproduction of pseudoscalar and longitudinally polarized vector mesons at moderately large photon virtuality. Although the soft physics approach also applies to large momentum transfer photoproduction of mesons it seems - as judged on the basis of the present data - that the contributions from the hadronic component of the photon dominate these reactions up to rather high energies. The kinematical region in which the soft physics approach is applicable to electroproduction, is accessible to experiments at the upgraded TJlab and at the proposed ELFE accelerator and EPIC collider. The measurement of large momentum transfer electroproduction of mesons is certainly difficult but seems feasible. We have not discussed electroproduction of flavored mesons and of transversally polarized vector mesons in this work. These processes involve flavor or helicity non-diagonal SPDs which are not directly related to those appearing in 
the processes we have investigated. As in DVEM [51, 25], higher twist dynamics plays an important role in electroproduction of transversally polarized vector mesons since the leading twist, lowest order subprocess amplitudes are zero in this case.

\section{Acknowledgment}

We would like to thank Markus Diehl, Thorsten Feldmann and Rainer Jakob for many stimulating discussions. H.W. Huang thanks the Deutsche Forschungsgemeinschaft for support.

\section{References}

[1] D. Müller, D. Robaschik, B. Geyer, F.-M. Dittes and J. Hořejši, Fortschr. Physik 42, 101 (1994), hep-ph/9812448.

[2] X. Ji, Phys. Rev. Lett. 78, 610 (1997) hep-ph/9603249; Phys. Rev. D55, 7114 (1997) hep-ph/9609381.

[3] A.V. Radyushkin, Phys. Rev. D56, 5524 (1997) hep-ph/9704207.

[4] X. Ji and J. Osborne, Phys. Rev. D58, 094018 (1998) hep-ph/9801260; J.C. Collins and A. Freund, Phys. Rev. D59, 074009 (1999) [hep-ph/9801262].

[5] A.V. Radyushkin, Phys. Lett. B385, 333 (1996) hep-ph/9605431.

[6] J.C. Collins, L. Frankfurt and M. Strikman, Phys. Rev. D56, 2982 (1997) hep$\mathrm{ph} / 9611433$.

[7] M. Diehl, T. Feldmann, R. Jakob and P. Kroll, Eur. Phys. J. C8, 409 (1999), hepph/9811253.

[8] A.V. Radyushkin, Phys. Rev. D58, 114008 (1998) hep-ph/9803316.

[9] M. Diehl, T. Feldmann, R. Jakob and P. Kroll, Phys. Lett. B460, 204 (1999) hhepph/9903268.

[10] G.P. Lepage and S.J. Brodsky, Phys. Rev. D22, 2157 (1980).

[11] J. Gronberg et al., CLEO collaboration, Phys. Rev. D57, 33 (1998) hep-ex/9707031.

[12] R. Jakob, P. Kroll and M. Raulfs, J. Phys. G22, 45 (1996) hep-ph/9410304.

[13] I. V. Musatov and A. V. Radyushkin, Phys. Rev. D56, 2713 (1997), [hep-ph/9702443].

[14] P. Kroll and M. Raulfs, Phys. Lett. B387, 848 (1996) hep-ph/9605264. 
[15] J. Bolz, R. Jakob, P. Kroll, M. Bergmann and N.G. Stefanis, Z. Phys. C66, 267 (1995) hep-ph/9405340.

[16] J. Bolz and P. Kroll, Z. Phys. A356, 327 (1996) hep-ph/9603289.

[17] N. Isgur and C.H. Llewellyn Smith, Nucl. Phys. B317, 526 (1989); A.V. Radyushkin, Nucl. Phys. A532, 141c (1991).

[18] J. Botts and G. Sterman, Nucl. Phys. B325, 62 (1989); H.-N. Li and G. Sterman, Nucl. Phys. B381, 129 (1992).

[19] T. Brooks and L. Dixon, hep-ph/0004143.

[20] M.A. Shupe et al., Phys. Rev. D19, 1921 (1979).

[21] G.R. Farrar, K. Huleihel and H. Zhang, Nucl. Phys. B349, 655 (1991).

[22] R.L. Anderson et al., Phys. Rev. D14, 679 (1976).

[23] M. Diehl and T. Gousset, Phys. Lett. B428, 359 (1998) hep-ph/9808479.

[24] L. Mankiewicz, G. Piller and T. Weigl, Eur. Phys. J. C5, 119 (1998) hep-ph/9711227;

M. Vanderhaeghen, P. A. Guichon and M. Guidal, Phys. Rev. D60, 094017 (1999) hepph/9905372].

[25] L. Mankiewicz and G. Piller, Phys. Rev. D61, 074013 (2000) hep-ph/9905287.

[26] S. J. Brodsky, L. Frankfurt, J. F. Gunion, A. H. Mueller and M. Strikman, Phys. Rev. D50, 3134 (1994) hep-ph/9402283; L. Frankfurt, W. Koepf and M. Strikman, Phys. Rev. D54, 3194 (1996) hep-ph/9509311; J. Nemchik, N. N. Nikolaev and B. G. Zakharov, Phys. Lett. B341, 228 (1994) hep-ph/9405355; A. V. Radyushkin, Phys. Lett. B385, 333 (1996) hep-ph/9605431; P. Hoodbhoy, Phys. Rev. D56, 388 (1997) hep-ph/9611207.

[27] A. D. Martin, M. G. Ryskin and T. Teubner, Phys. Rev. D55, 4329 (1997) hepph/9609448 and hep-ph/9912551.

[28] J.B. Kogut and D.E. Soper, Phys. Rev. D1, 2901 (1970).

[29] M. Vanttinen and L. Mankiewicz, Phys. Lett. B434, 141 (1998) hep-ph/9805338.

[30] T. Feldmann, P. Kroll and B. Stech, Phys. Rev. D58, 114006 (1998) hep-ph/9802409.

[31] S.D. Drell and T.-M. Yan, Phys. Rev. Lett. 24, 181 (1970).

[32] M. Diehl, T. Feldmann, R. Jakob and P. Kroll, WU-B 00-10, in preparation

[33] B. Chibisov and A. R. Zhitnitsky, Phys. Rev. D52, 5273 (1995) hep-ph/9503476. 
[34] S. J. Brodsky, hep-ph/9807212.

[35] M. Glück, E. Reya and A. Vogt, Z. Phys. C67, 433 (1995) and Eur. Phys. J. C5, 461 (1998) hep-ph/9806404; M. Glück, E. Reya, M. Stratmann and W. Vogelsang, Phys. Rev. D53, 4775 (1996) hep-ph/9508347.

[36] V. Y. Petrov, M. V. Polyakov, R. Ruskov, C. Weiss and K. Goeke, Phys. Rev. D59, 114018 (1999) hep-ph/9807229]; I. V. Anikin, A. E. Dorokhov and L. Tomio, hep$\mathrm{ph} / 9909368$.

[37] V. M. Braun, A. Khodjamirian and M. Maul, Phys. Rev. D61, 073004 (2000) hep$\mathrm{ph} / 9907495)$.

[38] T. Feldmann and P. Kroll, Eur. Phys. J. C5, 327 (1998) hep-ph/9711231.

[39] P. Ball and V. Braun, Phys. Rev. D54, 2182 (1996) hep-ph/9602323.

[40] M. Neubert and B. Stech, hep-ph/9705292.

[41] S.J. Brodsky and G.R. Farrar, Phys. Rev. Lett. 31, 1153 (1973); V.A. Matveev, R.M. Muradyan and A.N. Tavkhelidze, Lett. Nouvo Cim. 7, 719 (1973).

[42] C. F. Berger and W. Schweiger, hep-ph/9910509.

[43] T. Buran et al., Nucl. Phys. B111, 1 (1976).

[44] J.-M. Laget, hep-ph/0003213.

[45] J.J. Sakurai, Currents and Mesons, the University of Chicago Press, Chicago (1960).

[46] I. F. Ginzburg and D. Y. Ivanov, Phys. Rev. D54, 5523 (1996) hep-ph/9604437; D. Y. Ivanov, R. Kirschner, A. Schafer and L. Szymanowski, Phys. Lett. B478, 101 (2000) hep-ph/0001255.

[47] J.D. Bjorken and E.A. Paschos, Phys. Rev. 185, 1975 (1969); D.M. Scott, Phys. Rev. D10, 3117 (1974).

[48] P. Kroll, M. Schurmann and P. A. Guichon, Nucl. Phys. A598, 435 (1996) hep$\mathrm{ph} / 9507298$.

[49] J. Breitweg et al., ZEUS collaboration, Eur. Phys. J. C6, 603 (1999).

[50] A. M. Nathan, E97-108 collaboration, hep-ph/9807397.

[51] M. Diehl, T. Gousset and B. Pire, Phys. Rev. D59, 034023 (1999) hep-ph/9808479. 